\title{
NO/cGMP signalling
}

\section{Doris Koesling*, Evanthia Mergia, Michael Russwurm, Florian Mullershausen, Corina Wagner, Alexander Lange and Andreas Friebe}

\author{
Address: Institut für Pharmakologie, Ruhr-Universität Bochum, 44780 Bochum, Germany. \\ Email: Doris Koesling* - koesling@iname.com \\ * Corresponding author
}

from 2nd International Conference of cGMP Generators, Effectors and Therapeutic Implications Potsdam, Germany, 10-12 June, 2005

Published: 16 June 2005

BMC Pharmacology 2005, 5(SuppI I):SI0 doi:I0.II86/I47I-22I0-5-SI-SI0

Most of the effects of the signalling molecule nitric oxide (NO) are mediated by the stimulation of the NO-sensitive guanylyl cyclase (GC) and the subsequent increase in cGMP formation. The enzyme contains a prosthetic heme group which mediates NO stimulation. In addition to the physiological activator NO, NO-sensitisers like the substance YC- 1 sensitise the enzyme towards NO and may therefore have important pharmacological implications. Two isoforms of NO-sensitive GC have been identified to date that share regulatory properties but differ in the subcellular localization; the more ubiquitously expressed $\alpha_{1} \beta_{1}$ heterodimer and the $\alpha_{2} \beta_{1}$ isoform mainly expressed in brain. Knock-out mice in which either one the GC subunits is deleted will provide information about the functional roles of GC and the isoforms.

In intact cells, NO-induced cGMP signalling not only depends on cGMP formation but is also critically determined by the activity of the enzyme responsible for cGMP degradation i.e. phosphodiesterase 5 (PDE5). Recently, direct activation of PDE5 by cGMP was demonstrated which is limiting the cGMP increase and therefore is functioning as a negative feedback. As the cGMP-induced PDE5 activation turned out to be sustained, in the range of hours, it is probably responsible for the NO-induced desensitisation observed within NO/cGMP signalling. 